\title{
Dificultades con la adherencia al tratamiento no farmacológico de pacientes con falla cardiaca detectados a través de seguimiento telefónico'
}

\author{
Edith del Socorro Arredondo Holguín² \\ María de los Ángeles Rodríguez Gázquez ${ }^{3}$ \\ Lina Marcela Higuita Urrego ${ }^{4}$
}

doi:10.11144/Javeriana.IE16-2.dcat

Cómo citar: Arredondo Holguín ES, Rodríguez Gásquez MA, Higuita Urrego LM. Dificultades con la adherencia al tratamiento no farmacológico de pacientes con falla cardiaca detectados a través de seguimiento telefónico. Investig Enferm. Imagen Desarr. 2014;16(2):133-147. doi:10.11144/Javeriana.IE16-2.dcat

1. Artículo de investigación. Recibido 17 de marzo de 2014. Evaluado: 29 de abril a 7 de junio de 2014. Aceptado: 1 de julio de 2014. Derivado del proyecto de investigación Impacto de un programa educativo en enfermeria en la mejoria de los comportamientos de autocuidado en pacientes con falla cardiaca en una institución hospitalaria de Medellín, financiado por la Universidad de Antioquia.

2. Enfermera. Magíster en Enfermería. Profesora de la Facultad de Enfermeria, Universidad de Antioquia, Medellín, Colombia. Correo electrónico: tapua412@tone.udea.edu.co, edith. arredondo@udea.edu.co

3. Enfermera. Epidemióloga y doctora en Salud Pública. Profesora de la Facultad de Enfermería de la Universidad de Antioquia, Medellín, Colombia. Correo electrónico: mariangelesrodriguezq@hotmail.com

4. Enfermera de la Facultad de Enfermería de la Universidad de Antioquia, Medellín, Colombia. Correo electrónico: linamarcela-17@hotmail.com 


\section{Resumen}

Objetivo: Describir las dificultades detectadas en el seguimiento telefónico para la práctica de comportamientos de autocuidado relacionadas con la adherencia al tratamiento no farmacológico en pacientes con falla cardiaca (FC). Metodología: Investigación descriptiva en la que participaron 31 pacientes con $\mathrm{FC}$ de un hospital de Medellín, Colombia, en 2011. Se realizaron 7 sesiones mensuales de seguimiento telefónico a los pacientes. En cada sesión (30-45 minutos) se evaluaron las dificultades con la adherencia a 9 conductas de autocuidado. Todos los participantes recibieron educación en enfermería según sus necesidades individuales. Resultados: Todos los pacientes tenían dificultades en la práctica de algunas actividades de autocuidado relacionadas con la adherencia al tratamiento no farmacológico, el 83,9\% de ellos para 5 y más actividades. Las conductas que tenían más dificultades fueron: medir la orina y controlar el peso (100\% en cada una), el control de consumo de sal (96,7\%) y la restricción de líquidos (93,5\%). Conclusión. Con la ayuda del seguimiento telefónico se identificó la educación impartida por el personal de salud como la principal dificultad en la adherencia al tratamiento no farmacológico en los pacientes con FC. Los aspectos individuales fueron importantes en el control del consumo de sal, disminución de las actividades diarias y en la reducción del consumo de alcohol y cigarrillo. Para el control del peso el aspecto más referido fue de carácter social.

Palabras clave: insuficiencia cardiaca; autocuidado; educación en enfermería; cooperación del paciente

\section{Difficulties with Adherence to Non-Pharmacological Treatment of Patients with Heart Failure Detected through Telephone Follow-up}

\section{Abstract}

Objective: To describe the difficulties encountered in the telephone follow-up for the practice of self-care behaviors related to adherence to non-pharmacological treatment in patients with heart failure (HF). Methodology: Descriptive study in which 31 patients with HF of a hospital in Medellin, Colombia, in 2011 participated.7 per month telephone follow-up sessions for patients were conducted. Each session (30-45 minutes) difficulties with adherence to 9 self-care behaviors were assessed. All participants received nursing education according to their individual needs. Results: All patients had difficulties in practicing some self-care activities related to non-adherence to drug therapy, $83.9 \%$ of them for 5 or more activities. The behaviors that were more difficult were: measuring urine and weight control (100\% each), control of salt intake $(96.7 \%)$ and fluid restriction $(93.5 \%)$. Conclusion: With the help of telephone follow-up education given by health personnel was identified as the main difficulty in regards to the adherence to non-pharmacological treatment in patients with HF. Individual issues were important in the control of salt intake, decreased daily activities and the reduction of alcohol consumption and cigarette. For weight control the most reported aspect was of social nature.

Keywords: heart failure; self-care; nursing education; patient cooperation 


\section{Dificuldades com a adesão ao tratamento não- farmacológico entre pacientes com insuficiência cardiaca detectada por meio de contato telefónico}

\section{Resumo}

Objetivo: Descrever as dificuldades detectadas no contato telefónico para a prática de comportamentos de autocuidado relacionadas com a adesão ao tratamento nãofarmacológico entre pacientes com insuficiência cardíaca (IC). Metodologia: Pesquisa descritiva em que participaram 31 pacientes com IC de um hospital de Medellin, Colômbia, em 2011. Foram realizadas 7 sessões mensais de seguimento por meio de contato telefónico aos pacientes. Em cada sessão (30-45 minutos) foram avaliadas as dificuldades com a adesão para 9 condutas de autocuidado. Todos os participantes receberam instrução em enfermagem de acordo com as necessidades individuais. Resultados: Todos os pacientes tinham dificuldades na prática de algumas atividades de autocuidado relacionadas com adesão ao tratamento não-farmacológico, 83,9\% deles para 5 e más atividades. As condutas que tinham maiores dificuldades foram: medir a urina e controlar o peso ( $100 \%$ em cada uma), o controle no consumo de sal $(96,7 \%)$ e a restrição de líquidos $(93,5 \%)$. Conclusão: Com a ajuda de seguimento telefónico identificou-se a instrução ministrada pelo pessoal de saúde como a principal dificuldade na adesão ao tratamento não-farmacológico em pacientes com IC. Os aspectos individuais foram importantes no controle de consumo de sal, diminuição das atividades diárias e na redução de consumo de álcool e cigarro. Para o controle do peso o aspecto mais referido foi de caráter social.

Palavras-chave: insuficiência cardíaca; autocuidado; instrução em enfermagem; cooperação do paciente. 


\section{Introducción}

En el mundo, la falla cardiaca (FC) es considerada un grave problema de salud pública debido a su altísima morbilidad y mortalidad (1) y por los enormes costos económicos y sociales que genera a los pacientes, a sus familias, a los proveedores de servicios de salud y a la sociedad en general (2). La fatiga extrema que sufren las personas con esta enfermedad es causada por la baja perfusión en los tejidos corporales, lo cual influye en el deterioro de la calidad de vida, afecta los roles personal y social y las lleva a la pérdida progresiva de la capacidad de cuidarse (3). Por lo anterior, uno de los principales retos para enfermería es mejorar el autocuidado de la persona con FC (4).

El autocuidado fue definido por Orem (5) como la práctica de conductas que las personas hacen por sí mismas de manera consciente y permanente para el mantenimiento de la vida, el desarrollo, la salud y el bienestar, pues permiten que cuando se hacen eficazmente, contribuyan de forma específica a la integridad estructural, funcionamiento y desarrollo humano. Uno de cada diez pacientes con enfermedades crónicas tiene dificultades con la adherencia al tratamiento no farmacológico (6), los cuales están relacionados principalmente con conductas inadecuadas de salud como el tabaquismo, la obesidad y el sedentarismo. Estas conductas son susceptibles de cambiar mediante la utilización de estrategias educativas que mejoren el conocimiento y que incidan en la autogestión y manejo de la enfermedad (7-9).

La llamada telefónica es una de las tecnologías de la telecomunicación más accesibles que tiene la teleenfermería (10) para reforzar el cuidado a los pacientes cuando se encuentran en casa $(11,12)$. Se ha visto que los programas educativos de enfermería dirigidos a pacientes que emplean la estrategia de seguimiento telefónico por periodos superiores a tres meses han sido efectivos en el mejoramiento de la adherencia terapéutica $(13,14)$, lo cual redunda en un mejor control de la enfermedad, comparado con la atención ordinaria $(15,16)$. Este artículo pretende describir las dificultades detectadas en el seguimiento telefónico para la práctica de comportamientos de autocuidado, relacionadas con la adherencia al tratamiento no farmacológico en pacientes con FC.

\section{Metodología}

Investigación descriptiva en la que participaron 31 pacientes con $\mathrm{FC}$ de una institución hospitalaria de la ciudad de Medellín en 2011. Cada paciente recibió 7 sesiones de seguimiento telefónico, con frecuencia de una mensual.

Cada sesión fue planeada para evaluar los avances o dificultades que presentaban los pacientes con las conductas de autocuidado y brindar la educación en enfermería de acuerdo con las necesidades individuales de la persona. Las sesiones fueron realizadas por una investigadora principal y cuatro estudiantes de enfermería de último semestre, quienes fueron estandarizados en la valoración de las conductas de autocuidado relacio- 
nadas con la adherencia no farmacológica y la educación en enfermería que deberían brindar por teléfono a los participantes del estudio.

Los pacientes fueron agendados para el seguimiento mensual desde el momento de ingreso al estudio. Cada llamada telefónica de seguimiento tuvo una duración de entre 30 y 45 minutos y se realizaron en horarios diurnos; cuando por alguna razón no era encontrado el paciente, se siguió insistiendo durante los días siguientes hasta lograr el contacto. En ocasiones, durante la sesión del seguimiento telefónico los alumnos tuvieron dudas respecto a la educación que deberían brindar por el estado de salud del paciente, por lo que la investigadora pasó al teléfono y evaluó la situación y terminó de dar la educación. En todos los casos en los que se identificó que el paciente se estaba descompensando, se aconsejó la pronta consulta a un médico. Con frecuencia, durante las sesiones de seguimiento telefónico, los cuidadores aclararon a la enfermera la situación del paciente y resolvieron dudas sobre el cuidado de la persona en casa.

Para recolectar la información se utilizó una ficha que contenía las variables demográficas (edad, sexo, estado civil, ocupación y nivel de estudios), de apoyo social (familia, amigos e institución de salud) y clínicas (comorbilidad, clase funcional, fracción de eyección, hospitalizaciones y muerte). Con excepción de los datos clínicos extraídos de la historia clínica en la institución de salud, el resto de la información fue obtenida directamente del paciente.

También se empleó un formato para las sesiones de seguimiento telefónico que contenía un listado con los comportamientos de autocuidado relacionados con la adherencia no farmacológica: control de peso, medición de la orina, restricción de líquidos, control del consumo de sal, realización de actividad física, restricción del consumo de alcohol y tabaco, elevación de los pies y distribución de las actividades diarias (17), los cuales eran verificados en cada sesión respecto a su práctica con la información suministrada por el paciente. Se anotaron en la casilla de observaciones de enfermería las dificultades para la práctica de los comportamientos de autocuidado, los avances en el cuidado después de la última sesión y las recomendaciones suministradas por la enfermera para la mejoría de la situación encontrada.

Por último, las investigadoras evaluaron las dificultades de autocuidado, quienes las agruparon según fueran atribuibles a la enfermedad o a aspectos individuales, sociales o educativos del paciente (18). La información recolectada se analizó con el programa SPSS versión 19 (Chicago, Estados Unidos). La descripción general del grupo de estudio se hizo con proporciones para las variables categóricas y se calcularon la mediana, el rango intercuartílico y los valores mínimo y para las variables cuantitativas. A las dificultades para el autocuidado se les estimaron proporciones.

Esta investigación recibió el aval del Comité Técnico de Investigación de la Facultad de Enfermería de la Universidad de Antioquia. Los principales aspectos éticos tenidos en cuenta fueron: el consentimiento informado firmado y la confidencialidad en la información tomada para la investigación. 


\section{Resultados}

Fueron objeto de seguimiento telefónico 31 personas con FC que cumplieron con los criterios de inclusión. Las características generales de este grupo son las siguientes: personas con una mediana de edad de 62 años (rango intercuartílico: 58-72; mínimo: 30; máximo: 83), un 54,2\% son mujeres y 45,8\% son hombres; por estado civil predominaron los casados o en unión libre (61,3\%), siguiéndoles los viudos $(19,4 \%)$, los solteros $(16,1 \%)$ y los separados (3,2\%). Por estrato socioeconómico, el 87,1\% de los participantes pertenecía al 3 o a uno menor, y el 12,9\%, al 4 o a uno superior. Un $80,1 \%$ tiene estudios primarios, y el resto $(19,1 \%)$, secundarios o más altos. La principal actividad de los pacientes eran las labores del hogar $(58,1 \%)$, seguida por el trabajo remunerado $(6,5 \%)$ y las actividades recreativas (9,7\%); un $25,8 \%$ no realizaba ninguna actividad. Recibian apoyo principalmente de la familia $(80,6 \%)$, de las instituciones de salud $(35,5 \%)$ y de los amigos (16,1\%). Un 9,7\% no tenía ningún tipo de apoyo social.

Con respecto a las variables clínicas, según la clasificación funcional de la New York Hearth Association, el 83,9\% pertenecía a una clase funcional mayor de I (II: 38,7\% y III: 45,2\%) y el resto era clase I $(16,1 \%)$. E1 $66,7 \%$ tenía una fracción de eyección por debajo del $50 \%$ y presentaba como principales comorbilidades la hipertensión arterial $(83,9 \%)$, la enfermedad coronaria $(35,5 \%)$, la diabetes mellitus $(32,3 \%)$ y la insuficiencia renal crónica (22,6\%). Durante el periodo de estudio, 9 de cada 10 pacientes fueron hospitalizados, siendo la mediana del número de internaciones de uno.

Todos los participantes de esta investigación que recibieron seguimiento telefónico tenían dificultades en la práctica de algunas conductas de autocuidado relacionadas con la adherencia al tratamiento no farmacológico: el 83,9\% de ellos para 5 y más conductas de las 9 priorizadas para la educación en este estudio. En la tabla 1 se evidencia que las actividades con más dificultades para los pacientes fueron: medir la orina y controlar el peso (100\% en cada una), el control de consumo de sal $(96,7 \%)$ y la restricción de líquidos $(93,5 \%)$.

TABLA 1. Proporción de pacientes con falla cardiaca con dificultades en la adherencia a actividades del tratamiento no farmacológico $(n=31)$

\begin{tabular}{|l|r|}
\hline \multicolumn{1}{|c|}{ Actividad } & \multicolumn{1}{c|}{$\%$} \\
\hline Control del consumo de sal & 51,6 \\
\hline Medición de la orina & 100,0 \\
\hline Control del peso & 100,0 \\
\hline Restricción de líquidos & 93,5 \\
\hline Prevención de la gripe & 83,9 \\
\hline Realizar actividad física & 83,9 \\
\hline Distribución de las actividades diarias & 51,4 \\
\hline Levantar los piernas 30 grados & 64,5 \\
\hline Reducción del consumo de alcohol y tabaco & 16,1 \\
\hline
\end{tabular}

Fuente: datos de la información recolectada en este estudio. 
Los factores a los que se atribuyeron las dificultades con la práctica de actividades de autocuidado en los pacientes con FC pueden ser observadas en la tabla 2 . El factor educativo fue reportado con mayor frecuencia en las actividades de medir la orina, restricción de líquidos, prevención de la gripe y levantar los pies; mientras que el individual fue el más importante en el control del consumo de sal, la disminución de las actividades diarias y en la reducción del consumo de alcohol y tabaco. Para el control del peso el factor más referido fue de carácter social.

TABLA 2. Factor atribuible a la dificultad con la práctica de actividades de autocuidado relacionadas con la adherencia no farmacológica en 31 pacientes con falla cardiaca

\begin{tabular}{|l|c|c|c|c|}
\hline \multirow{2}{*}{\multicolumn{1}{|c|}{$\begin{array}{c}\text { Actividad de } \\
\text { autocuidado }\end{array}$}} & \multicolumn{4}{|c|}{ Factor atribuible* } \\
\cline { 2 - 5 } & $\begin{array}{c}\text { Enfermedad } \\
\text { (\%) }\end{array}$ & $\begin{array}{c}\text { Individual } \\
\text { (\%) }\end{array}$ & $\begin{array}{c}\text { Social } \\
\text { (\%) }\end{array}$ & $\begin{array}{c}\text { Educativo } \\
\text { (\%) }\end{array}$ \\
\hline $\begin{array}{l}\text { Control del consumo de } \\
\text { sal (n = 16) }\end{array}$ & 0,0 & 83,3 & 13,3 & 23,4 \\
\hline Medir orina (n = 31) & 9,6 & 22,6 & 6,5 & 87,1 \\
\hline Control del peso (n=31) & 19,4 & 22,6 & 54,8 & 45,2 \\
\hline $\begin{array}{l}\text { Restricción de líquidos } \\
\text { (n=29) }\end{array}$ & 13,8 & 31,0 & 13,8 & 79,3 \\
\hline $\begin{array}{l}\text { Prevención de la gripe } \\
\text { (n=26) }\end{array}$ & 7,8 & 30,8 & 19,2 & 61,5 \\
\hline Actividad física (n=26) & 69,3 & 30,8 & 30,8 & 0,0 \\
\hline $\begin{array}{l}\text { Distribución de las activi- } \\
\text { dades diarias (n = 16) }\end{array}$ & 31,2 & 50,0 & 31,2 & 6,2 \\
\hline $\begin{array}{l}\text { Levantar los piernas 30 } \\
\text { grados (n = 20) }\end{array}$ & 25,0 & 5,0 & 15,0 & 64,9 \\
\hline $\begin{array}{l}\text { Reducción del consumo de } \\
\text { alcohol y tabaco (n = 5) }\end{array}$ & 0,0 & 80,0 & 0,0 & 20,0 \\
\hline
\end{tabular}

* Una persona en cada actividad de autocuidado puede tener más de una factor atribuible que produce dificultad para su práctica.

FUENTE: datos de la información recolectada en este estudio

A continuación se presentan algunos de los comentarios de los pacientes relacionados con cada una de las actividades de autocuidado en la cual presentaron dificultades para su adherencia.

\section{Control del consumo de sal}

Los pacientes comentan principalmente motivos individuales: "no he sido capaz, me da mucha dificultad", "la sal me gusta mucho". Los aspectos educativos que más relataron son los asociados a la falta de información suministrada por el equipo de salud: "No me han limitado la sal", "controlo 
la sal un poquito". Algunos participantes mostraron tener dificultades con aspectos de adhesión a la dieta, que son muy importantes en el manejo de su condición, como son la cantidad y el tipo de alimentos consumidos: "controlo la sal, pero no los dulces y como mucho", además de que otros pacientes se han acostumbrado a una adherencia incompleta: "solo por momentos sigo la dieta”. Los que dicen que saben manejar su dieta informan que lo aprendieron especialmente de la nutricionista, están convencidos de la importancia de este autocuidado y lo han hecho parte de la vida diaria: "comer bien se ha vuelto costumbre". De las causas sociales, la dificultad económica es la más representativa: "es muy difícil cuidarme en la dieta, pues no tengo mucho que comer", que habla de un bajo acceso a los alimentos de algunos de los pacientes.

\section{Medir la orina}

Algunos pacientes tuvieron causas físicas que justificaron la no realización de esta actividad, como la asistencia a sesiones de hemodiálisis o que estuvieron internados por cirugía cardiaca o por complicaciones de su estado de salud; sin embargo, estos últimos manifestaron que después de la hospitalización tampoco medían la orina.

Los pacientes también manifestaron tener razones para no practicar la medición de orina, las cuales se pueden reunir dentro del grupo de causas individuales, que van desde que orinan mucho a que lo hacen muy poco. Otros creen que no es necesario realizar esta práctica, se les olvida o no les gusta hacerlo, pues les parece difícil realizarla. Esta última razón, junto con las que siguen de ejemplo, da cuenta de que la falta de educación impartida por el personal de salud es una causa importante para la no adherencia a esta actividad: "lo hago cuando estoy hinchado; de resto, no", "a mí no me habían dicho que era necesario" o "no sé medir la orina".

\section{Control del peso}

Algunos pacientes comentaron no poder pesarse por su mal estado general: "no me puedo tener en pie", y a otros se les olvida. Uno de los motivos más frecuentes es la falta de pesa, que no puede comprar o mandar a arreglar, por no disponer de dinero. La falta de educación en salud es un motivo importante que se refleja en las expresiones: "solo me peso cuando voy al médico", "el cardiólogo no me informó", "lo controlo poco; no sé para qué" o "no me dijeron".

\section{Restricción de líquidos}

140 Los participantes comentan razones físicas asociadas a su enfermedad y comorbilidades existentes a la dificultad de controlar los líquidos: "me da 
mucha sed"; mientras que otros lo asumen para evitar complicaciones: "tomar líquido me hace daño". Los motivos individuales para no controlar los líquidos ingeridos incluyen razones como: "no le hago caso al doctor", "se me olvida", "el médico me dijo que disminuyera el líquido y no lo hago; soy muy tranquila" y "tengo vaso para medir pero no lo hago". También dicen no hacerlo cuando están trabajando, ni cuando salen a la calle. Los motivos asociados a la falta de educación en salud en este tema coinciden con los informados en las actividades de autocuidado: "no sé para qué se hace", "no sé cuánta cantidad puedo tomar" y "nunca me han dicho que debo restringir los líquidos".

\section{Prevención de la gripe}

En general, los pacientes no se vacunan. Ni siquiera uno de ellos que dijo haber sufrido de neumonía por el virus H1N1. Entre las razones que se pueden agrupar como individuales se encuentran: "no me cuido de la gripa", "me han dado muchas gripas complicadas y no me he vacunado", "me dan miedo las vacunas", "nunca me vacuno", "se me olvida", "me da pereza", "no me parece importante", que son además evidencia de dificultades en la educación en salud que han recibido. Algunos participantes, aunque no se vacunan contra la gripe, manifiestan que se cuidan de exponerse a personas que tengan gripe; para otros, es difícil el aislamiento, pues viven con niños o comparten una vivienda pequeña con otras personas.

\section{Actividad física}

La actividad física que más realizan los participantes en este estudio es caminar, la cual es combinada con las tareas del hogar cuando los pacientes se sienten mejor. Los que no practican actividad física generalmente comentan motivos relacionados con la enfermedad: "antes tenía oxígeno, me da miedo por el corazón", "camino poco, pues apenas mi corazón se está recuperando", "después de la cirugía no soy capaz", "me asfixio", "me da dolor en el corazón" o "no lo hago mucho, pues no puedo salir solo". Otras razones son individuales: "me da pereza", "no tengo tiempo, y soy muy floja”; algunos caminan cuando van al trabajo, mientras que otros no lo pueden hacer porque el lugar en el que viven está en un lugar muy escarpado, hay difíciles condiciones de inseguridad en su sector de residencia y faltan centros recreativos para adultos.

\section{Distribución de las actividades diarias}

Los pacientes comentan que lo hacen cuando tienen sintomas o cuando dependen del oxígeno; otros dicen no poder realizarlo, porque no tienen quién los ayude con las tareas domésticas. Los pacientes intentan descansar 
en cama tres veces al día y después de las actividades que les generan esfuerzo; los que trabajan, lo hacen sentados.

\section{Levantar las piernas 30 grados cuando se sienta}

Es una de las actividades con menor adherencia. La principal dificultad es que no se acuerdan o no sabian que lo tenían que hacer regularmente. Solo practican esta actividad cuando se les edematizan las piernas.

\section{Restricción del consumo de alcohol y tabaco}

En el grupo de estudio fue bajo el consumo de estas sustancias: tres personas fumaban (dos de ellas refirieron haber reducido la cantidad diaria de cigarrillos) y cuatro no controlan el consumo de alcohol. En todos los casos la familia los ha apoyado en la reducción del consumo.

\section{Discusión}

En este estudio de 31 pacientes con FC que fueron objeto de seguimiento telefónico mensual durante 7 meses, todos tenían dificultades en la práctica de algunas de las actividades de autocuidado relacionadas con la adherencia al tratamiento no farmacológico. Fueron las dificultades más frecuentes las que tuvieron que ver con la medición de la orina, el control de peso y las restricciones en el consumo de sal y de líquidos; todas están relacionadas con el riesgo de que paciente acumule líquidos y desarrolle complicaciones debidas a su FC (19).

Las conductas relacionadas con la adaptación a la enfermedad, como la distribución de las actividades diarias y el levantar las piernas 30 grados fueron inadecuadas en uno de cada dos pacientes. Estos comportamientos de autocuidado son esenciales para disminuir sintomas como la sensación de ahogo, el edema y la fatiga, y deben ser planeadas para satisfacer las necesidades básicas y de ocio (20). También en la sesiones de seguimiento telefónico se encontraron falencias en el control de otros comportamientos de autocuidado, como la realización de actividad física y la prevención de la gripe (en cuatro de cada cinco pacientes para cada actividad) y en la reducción del consumo de alcohol y tabaco (uno de cada cinco pacientes).

Se observó en los participantes de la investigación que la falta de educación en salud fue una importante causa de dificultad en la realización de ocho de nueve comportamientos de autocuidado que se trabajaron en la sesiones de seguimiento telefónico, especialmente en medir la orina, restringir líquidos, levantar los pies y prevenir la gripe. Parte de los pacientes reportaron no haber sido informados por el personal de salud sobre la necesidad de su práctica o tenían conocimientos inadecuados sobre estos. Lo anterior está de acuerdo con lo reportado en un estudio de Camargo et al. (21), 
en el que el $76 \%$ de las personas con $\mathrm{FC}$ informó no haber recibido ningún tipo de educación acerca de su enfermedad, tratamiento y manejo. Ello llama la atención sobre el hecho de que una de las principales competencias que deben tener las enfermeras es la educación a los pacientes y a sus familias, con el fin incrementar el conocimiento y el empoderamiento de los pacientes en el manejo de la enfermedad, de manera que se generen conductas de autocuidado.

Los factores individuales para la falta de adherencia estuvieron especialmente presentes en los autocuidados de restricción del consumo de sal, disminución de las actividades diarias y reducción del consumo de alcohol y tabaco. Los pacientes mostraron que el conocimiento previo es insuficiente y que puede asociarse a su no práctica cuando comentaban motivos como la pereza o el olvido. Estos factores son susceptibles de cambio con la educación que intenta mejorar el conocimiento y los comportamientos que influyen en el deterioro de la salud (22).

Según McAlister et al. (15), la educación en salud es la vía más eficaz para el mejoramiento del autocuidado de las personas que viven con la FC, y por tal motivo Haynes et al. (6) sugerían hacer los esfuerzos que fueran necesarios para reconocer las dificultades que tenían los pacientes con el tratamiento, a fin de ayudarlos con la consejería a mantener la adherencia al régimen prescrito. Durante las sesiones telefónicas realizadas en este estudio, se observó el mejoramiento del interés de los pacientes por saber más de la enfermedad y por cambiar los comportamientos no adecuados, lo que Kim y Oh (23) han relacionado con una mejor adherencia terapéutica. Cuando los pacientes entienden el impacto beneficioso de los cambios en el comportamiento son más receptivos para realizarlos $(24,25)$; también en la capacidad física y en el bienestar emocional (26).

El factor social también fue responsable de las dificultades en la adherencia a las conductas de pesarse y medir la orina diariamente. Algunos pacientes no contaban con recursos económicos para la compra de una pesa o de un recipiente medidor de volumen, lo que disminuye la posibilidad de una detección temprana de síntomas que indiquen la necesidad de consultar a los servicios de salud (24). Lo anterior es de vital importancia, pues estos pacientes enfrentan el riesgo de complicaciones y muerte, si no cumplen con las recomendaciones de salud que les fueron prescritas. Haynes et al. (6) afirmaban que intervenir los factores de adherencia no farmacológica tiene mayor impacto en la morbilidad y mortalidad por FC, que cualquier innovación que pueda hacerse con un agente farmacológico específico.

Otros motivos relacionados con los factores sociales que fueron comentados por los participantes, especialmente para la adherencia a la actividad física son: la soledad, la inseguridad del sector de residencia, la falta de centros recreativos y las condiciones escarpadas de los barrios, lo cual tiene relación con lo que puntualizan Artinian et al. (27), de que las condiciones sociales de los pacientes influyen en la manera como ellos realizan actividades de autocuidado. 
Con frecuencia, en esta investigación los cuidadores pasaron al teléfono para aclarar asuntos relacionados con el cuidado de los pacientes. Este involucramiento ha sido identificado como un factor de éxito para la adherencia a conductas de autocuidado por varios autores $(6,19,21,28,29)$, y fue calificado por los participantes como de gran ayuda para la ejecución de las actividades de control de peso, ejercicio físico y disminución del consumo de alcohol y tabaco.

En el estudio, el uso del teléfono fue una buena estrategia para el seguimiento de la adherencia a las conductas de autocuidado y para el suministro de educación al paciente con FC. La educación debe propender al empoderamiento de estas personas, para que manejen adecuadamente su condición crónica y sean capaces de vigilar los cambios que lleven a complicaciones de su estado físico; por lo tanto, es indispensable que la enfermera encargada de realizar esta consejería al paciente y a su familia posea un adecuado conocimiento sobre el manejo de la enfermedad y que tenga habilidades de escucha que faciliten la fluidez de la comunicación (30).

Conociendo las necesidades físicas y emocionales del paciente con FC, la complejidad del tratamiento de la enfermedad y las falencias sociales y de acceso a los servicios de salud que se viven en Colombia, es un reto para las enfermeras impulsar programas educativos con estrategias pedagógicas útiles, capaces de lograr que las personas con FC tengan posibilidades de mejorar la calidad de vida, aun con la enfermedad, y que se comprometan a cuidar de sí mismos y a buscar ayuda cuando no lo puedan hacer.

\section{Conclusión}

La principal dificultad para la adherencia al tratamiento no farmacológico en los pacientes con FC es la relacionada con la educación impartida por el personal de salud, la cual fue referida en las actividades de medir la orina, restringir líquidos, prevenir la gripe y levantar los pies; mientras que el individual fue el más importante en controlar el consumo de sal, disminuir las actividades diarias y reducir el consumo de alcohol y tabaco. Para el control del peso el factor más referido fue de carácter social.

Se recomienda que en los programas educativos de enfermería a las personas con FC se incluya el seguimiento telefónico como estrategia para el mejoramiento de la adherencia al tratamiento.

\section{Referencias}

1. Albert N. Evidence-based nursing care for patients with heart failure. AACN Adv Crit Care. 2006;17(2):170-83.

2. Achury DM. Autocuidado y adherencia en pacientes con falla cardiaca. Aquichán [internet]. 2007 [citado 2014 Jun 18];7(2):139-60. Disponible en: http://www.scielo.org.co/scielo.php?script=sci arttext\&pid=S1657-59972007000200004\&lng=es. 
3. Scott L, Setter-Kliner K, Britton A. The effects of nursing interventions to enhance mental health and quality of life among individuals with heart failure. Appl Nurs Res [internet]. 2004 [citado 2014 jun 18];17(4):248-56. Disponible en: http://ac.els-cdn.com/ S0897189704000746/1-s2.0-S0897189704000746-main.pdf?_ tid=a6990780-f7ba-11e3-a54a-00000aacb362\&acdnat $=1403186858_{\text {_ }}$ c1addf2516c0a0cc43a2b28bf6018e7d.

4. Renpenning K, Taylor S. Self-care t theory of nursing: selected papers of Dorothea Orem. New York: Springer; 2003.

5. Orem D, Taylor SY, Renpenning K. Nursing: concepts of practice. 6th ed. Philadelphia: Mosby; 2001.

6. Haynes RB, McDonald HP, Garg AX. Helping patients follow prescribed treatment: clinical applications. JAMA [internet] 2002 [citado 2014 jun 18];288(22):2880-3. Disponible en: http://alignmap.com/ wp-content/uploads/basics/Helping \%20Patients \%20Follow \%20 Prescribed \%20Treatment \%20-\%20Clinical \%20Applications.pdf.

7. Evangelista L, Doering L, Dracup K, Hamilton M. Compliance behaviors of elderly patients with advanced heart failure. J Cardiovasc Nurs [internet]. 2003 [citado 2014 Jun 18];18(3):197-206. Disponible en: http://eurheartj.oxfordjournals.org/content/27/4/434.full.pdf.

8. Gheorghiade M, Zannad F. Modern management of acute heart failure syndrome. Eur Heart J [internet]. 2005 [citado 2014 jun 18];7(supp1 B):B3-7. Disponible en: http://eurheartjsupp.oxfordjournals.org/content/7/suppl_B/B3.full.pdf+htm1.

9. Baker DW, Asch SM, Keesey JW, Brown JA, Chan KS, Joyce G, et al. Differences in education, knowledge, self-management activities, and health outcomes for patients with heart failure cared for under the chronic disease model: the improving chronic illness care evaluation. J Card Fail [internet]. 2005 [citado 2014 jun 18];11(6):405-13. Disponible en: http://ac.els-cdn.com/S1071916405001375/1-s2.0S1071916405001375-main.pdf?_tid=64a0d14a-f7bb-11e3-946600000aacb360\&acdnat $=1403187177 \_6 c 8$ ef7fb4c6df48edcbdfe $7 d 6$ $9 \mathrm{e} 82978$.

10. Schlachta-Fairchild L, Elfrink V, Deickman A. Patient safety, telenursing, and telehealth. In: Hughes R. Patient safety and quality: An evidence-based handbook for nurses. Rockville: Agency for Healthcare Research and Quality; 2008 [citado 2014 Jun 18]. Disponible en: http://www.ahrq.gov/professionals/clinicians-providers/ resources/nursing/resources/nurseshdbk/nurseshdbk.pdf.

11. Astin F, Closs SJ, McLenachan J, Hunter S, Priestley C. The information needs of patients treated with primary angioplasty for heart attack: an exploratory study. Patient Educ Couns [internet]. 2008 [citado 2014 Jun 18];73(2):325-32. Disponible en: http://ac.els-cdn. com/S0738399108003236/1-s2.0-S0738399108003236-main.pdf?_ tid=aea5e53c-f7bb-11e3-ac5a-00000aacb35f\&acdnat $=1403187301$ 89f2aebd9cfd29a9bca7065ab07501f7. 
12. Gallagher R, McKinley S, Dracup K. Effects of a telephone counseling intervention on psychosocial adjustment in women following a cardiac event. Heart Lung [internet]. 2003 [citado 2014 jun 18];32(2):79-87. Disponible en: http://ac.els-cdn.com/ S014795630300027X/1-s2.0-S014795630300027X-main.pdf?_ tid=c8110862-f7bb-11e3-bccb-00000aacb35f\&acdnat $=1403187344$ ac55f1083dcbb4840a4f6569fcb793b2.

13. Smith CE, Dauz ER, Clements F, Puno FN, Cook et al. Telehealth services to improve nonadherence: a placebo-controlled study. Telemed J E Health. 2006;12(3):289-96.

14. Stolic S, Mitchell M, Wollin J. Nurse-led telephone interventions for people with cardiac disease: a review of the research literature. Eur J Cardiovasc Nurs [internet]. 2010 [citado 2014 jun 18];9(4):203-17. Disponible en: http://eprints.qut.edu.au/40201/1/40201.pdf.

15. McAlister F, S. S, Ferrua S, 2004 MJ. Multidisciplinary strategies for the management of heart failure patients at high risk for admission: A systematic review of randomized trials. J Am Coll Cardiol [internet]. 2004 [citado 2014 jun 18];44(4):810-9. Disponible en: http://ac.els-cdn.com/S0735109704011234/1s2.0-S0735109704011234-main.pdf?_tid=b52fcf56-f7bd-11e39821-00000aab0f6c\&acdnat $=1403188171$ fdb1b05626b6febdee5332b60eaecabd.

16. Wong FK, Mok MP, Chan T, Tsang MW. Nurse follow up of patients with diabetes: Randomized controlled trial. J Adv Nurs [internet]. 2004 [citado 2014 jun 18];50(4):391-402. Disponible en: http://onlinelibrary.wiley.com/doi/10.1111/j.1365-2648.2005.03404.x/pdf.

17. Jaarsma T, Stromberg A, Martenssen J, Dracup K. Development and testing of the European Heart Failure Self-Care Behaviour Scale. Eur J Heart Fail [internet]. 2003 [citado 2014 jun 18];5:363-70. Disponible en: http://www.researchgate.net/publication/7017911_ Development_and_testing_of_the_European_Heart_Failure_SelfCare_Behaviour_Scale.

18. Organización Mundial de la Salud, Organización Panamericana de la Salud. Adherencia a los tratamientos a largo plazo: pruebas para la acción [internet]. Washington: OPS; 2004 [citado 2014 Jun 18]. Disponible en: http://www.paho.org/hq/index.php?option=com docman\&task=doc_view\&gid=18722\&Itemid=.

19. Koelling TM, Johnson ML, Cody RJ, Aaronson KD. Discharge education improves clinical outcomes in patients with chronic heart failure. Circulation [internet]. 2005 [citado 2014 jun 18];111(2):17985. Disponible en: http://circ.ahajournals.org/content/111/2/179. full.pdf.

20. González B, Lupón J, Herreros J, Urrutia A, Altimir S, Coll R, et al. Patient's education by nurse: what we really do achieve? Eur J Cardiovasc Nurs. 2005;4(2):107-11. 
21. Camargo CM, Córdoba DN, Guio AM. La entrevista motivacional como intervención de enfermería para promover el autocuidado en pacientes con insuficiencia cardiaca en una institución de cuarto nivel en Bogotá, Colombia. Invest Enferm. Imagen Desarr [internet]. 2013 [citado 2014 jun 18];15(1):31-49. Disponible en: http://revistas.javeriana.edu. co/index.php/imagenydesarrollo/article/view/6023/4867.

22. Rankin S, Stallings K. Patient education, principles and practice. 4th ed. Philadelphia: Lippincott Williams and Wilkins; 2001.

23. Kim HS, Oh JA. Adherence to diabetes control recommendation: Impact of nurse telephone calls. J Adv Nurs [internet]. 2003;44(3):25661. Disponible en: http://onlinelibrary.wiley.com/doi/10.1046/ j.1365-2648.2003.02800.x/pdf.

24. Holst M, Willenheimer R, Martenson J, Lindholm M, Strömberg A. Telephone follow-up of self-care behaviour alter a single sesión education of patients with heart failure in primary health care. Eur J Cardiovasc Nurs [internet]. 2007 [citado 2014 JUN 18];6(2):153-9. Disponible en: http://cnu.sagepub.com/content/6/2/153.long.

25. López M, Rus C, Martínez MM, Cortez GA, Delgado M. Aplicación de un plan de cuidados de enfermería en un programa de insuficiencia cardiaca. Enferm Cardiol [internet]. 2007 [citado 2014 JUN 18];14(41):2532. Disponible en: http://www.enfermeriaencardiologia. com/revista/4102.pdf.

26. Whellan DJ, Gaulden L, Gattis WA, Granger B, Russell SD, Blazing MA, et al. The benefit of implementing a heart failure disease management program. Arch Intern Med [internet]. 2001 [citado 2014 jun 18];161(18):2223-8. Disponible en: http://archinte.jamanetwork. com/article. aspx?articleid=649163.

27. Artinian N, Morris M, Sloan M, Lange P. Self-care behaviors among patients with heart failure: issues in cardiovascular nursing. Heart Lung [internet]. 2002 [citado 2014 jun 18];31(3):161-72. Disponible en: http://www.sciencedirect.com/science/article/pii/ S0147956302059812.

28. Ajzan I. Attitudes, personality and behavior [internet]. New York: Open University Press; 2005 [citado 2014 Jun 18]. Disponible en: http://psicoexperimental.files.wordpress.com/2011/03/ajzeni-2005-attitudespersonality-and-behaviour-2nd-ed-open-university-press.pdf.

29. Achury D. Contexto clínico de la falla cardiaca. En: Adherencia al tratamiento en el paciente con falla cardiaca. Bogotá: Editorial Universidad Nacional de Colombia; 2007.

30. Webel AR, Okonsky J, Trompeta J, Holzemer WL. A systematic review of the effectiveness of peer-based interventions on health-related behaviors in adults. Am J Public Health [internet]. 2010 [citado 2014 jun 18];100(2):247-53. Disponible en: http://ajph.aphapublications.org/doi/full/10.2105/AJPH.2008.149419. 
